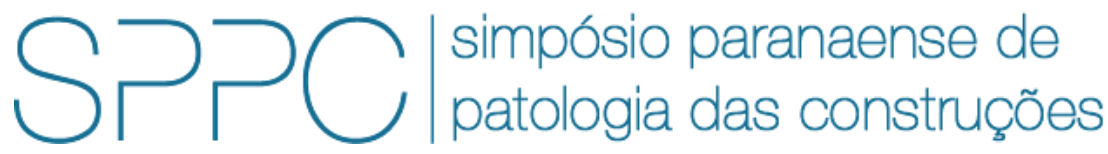

ISSN 2526-7248 artigo n. 2SPPC1012, pp. 129-143, 2017

\title{
Sensores de corrosão para monitoramento de pontes de Concreto armado - Testes iniciais
}

\author{
Analiet Calvo Valdés ${ }^{1}$, Polyana de Jesus Roque ${ }^{2}$ e M. H. F. Medeiros ${ }^{3}$ \\ ${ }^{1}$ Engenheira Mestranda, Universidade Federal do Paraná, analietcv@yahoo.es \\ 2Iniciação científica, Universidade Federal do Paraná, polyanaolegario@gmail.com \\ ${ }^{3}$ Professor Doutor, Universidade Federal do Paraná, medeiros.ufpr@gmail.com
}

\begin{abstract}
Resumo: A utilização de sensores embebidos no concreto para monitorar a durabilidade das estruturas é uma tendência atual. Uns dos dispositivos mais simples achados no mercado são os sensores galvânicos; formados por dois metais dissimilares onde um deles atua como ânodo e o outro como cátodo. O objetivo deste trabalho na sua primeira etapa foi tentar reproduzir nos laboratórios da UFPR um sensor galvânico de múltiplos elétrodos e avaliar o desempenho do mesmo em uma solução saturada de $\mathrm{Ca}(\mathrm{OH})_{2}$ com e sem adição de $\mathrm{NaCl}$ ao $3,5 \%$ em massa. O sensor projetado consiste em quatros barras retas de aço-carbono posicionadas em altura e ângulos diferentes sobre uma base de cobre com $50 \times 50 \mathrm{~mm}$ por $3 \mathrm{~mm}$ de espessura que atua como cátodo por ser este um metal com potencial eletroquímico mais estável do que o aço carbono. A altura dos ânodos com relação à superfície exposta do concreto é flexível e pode ser ajustada segundo a profundidade do recobrimento mínimo da estrutura. Durante o trabalho foi medida a corrente galvânica no circuito gerado entre os metais; em ambas as condições de exposição a corrente ficou da ordem de micros amperes. No intuito de inferir o potencial dos sensores foram avaliadas as leituras de diferença de potencial dos pares que os compõem; mas os sensores analisados não conseguiram manter ou voltar a um valor médio de potencial no transcurso do tempo.
\end{abstract}

Palavras-chaves: sensor, corrente galvânica, diferença de potencial.

Abstract: The use of sensors embedded in concrete to monitor the durability of structures is a current trend. Some of the simplest devices on the market are galvanic sensors; formed by two dissimilar metals where one of them acts as anode and the other as cathode. The objective of this work in its first stage was to try to reproduce in the laboratories of the UFPR a galvanic sensor of multiple electrodes and to evaluate its performance in a saturated solution of $\mathrm{Ca}(\mathrm{OH}) 2$ with and without addition of $\mathrm{NaCl}$ to $3,5 \%$ by weight. The designed sensor consists of four straight steel rods positioned at different angles and height on a square copper base with $50 \times 50 \mathrm{~mm}$ by $3 \mathrm{~mm}$ thickness that acts as a cathode because it is a metal with more stable electrochemical potential than steel carbon. The height of the anodes in relation to the exposed surface of the concrete is flexible and can be adjusted according to the depth of the minimum covering of the structure. During the work was measured the galvanic current in the circuit generated between the metals; in both conditions of exposure the current was of the order of micro amps. In order to infer the potential of the sensor were evaluated the potential difference readings of the pairs that compose it; but the sensors analyzed failed to maintain or return to an average potential value over time.

Keywords: sensor, galvanic current, potential difference. 
VALDÉS, A. C.; ROQUE, P. J.; MEDEIROS, M. H. F., SENSORES DE CORROSÃO PARA MONITORAMENTO DE PONTES DE CONCRETO ARMADO - TESTES INICIAIS. $2^{\circ}$ Simpósio Paranaense de Patologia das Construções (2० SPPC), artigo 2SPPC1012, pp. 129-143, 2017. DOI: $10.4322 / 2$ SPPC.2017.012

\section{Introdução}

A corrente galvânica aparece quando, em um mesmo meio condutivo, se faz o contato elétrico entre dois metais distintos ou entre dois metais similares, porém em estados distintos (estado ativo ou passivo) decorrentes das alterações do meio junto a um dos metais [1].

Em concreto íntegro, essa corrente é desprezível, ou seja, muito baixa. Isso porque as barras de aço-carbono estão em estado passivo, portanto, apresentam uma diferença de potencial desprezível em relação ao cátodo também passivo. O mesmo não ocorre quando um processo corrosivo é estabelecido nas barras. Nesse caso, a corrente galvânica apresenta um valor significativo em decorrência da variação do potencial do ânodo, que assume valores mais negativos em relação ao obtidos inicialmente, em seu estado passivo.

Enquanto a profundidade dos agentes agressores, diga-se, do dióxido de carbono que diminui o pH da solução de poros, ou dos íons cloretos em teores críticos não tiver atingido a posição do primeiro ânodo as correntes geradas entre os ânodos e o cátodo permanecem na gama das correntes típicas do estado passivo, isto é, menores do que $2 \mu \mathrm{A} / \mathrm{cm}^{2}$. Nesta fase, as variações que ocorrem no valor da corrente galvânica e do potencial de corrosão devem-se fundamentalmente às alterações das condições ambientais nomeadamente, temperatura, umidade do concreto e disponibilidade de oxigênio. À medida que os agentes agressivos vão penetrando na camada de recobrimento do concreto, a corrente galvânica se torna mais elevada, superior a $10 \mu \mathrm{A} / \mathrm{cm}^{2}$, e ocorre um decréscimo significativo do potencial de corrosão [2-3].

Contudo, nota-se que não há uma faixa fixa de valores de corrente galvânica que caracterizam o estado ativo das barras de aço-carbono do ânodo. Isso porque existe uma variedade de interferências na corrente circundante, como a área dos elétrodos, a qualidade do concreto e o teor de íons cloreto no material [4]. Assim sendo, não é o valor absoluto da corrente que deve ser considerado, mas sim a variação de seus valores ao longo do tempo [5].

A utilização de macro-pares galvânicos para o monitoramento da corrosão é um dos sistemas mais simples, pois a corrente galvânica gerada no circuito formado por dois metais pode ser detectada por um amperímetro de resistência nula e analisada para se obter dados sobre a probabilidade de corrosão na armadura de reforço.

Contrariamente aos tradicionais eletrodos de referência, os eletrodos galvânicos não estão baseados em reações termodinamicamente reversíveis, em lugar disso, consistem em pares bi metálicos que mantém um potencial estável por um período finito de tempo. A sua estabilidade é baseada no meio ambiente específico do concreto e pode ser explicado e calibrado com teoria eletroquímica [6].

Na prática, para a avaliação do risco de corrosão nas estruturas de concreto, os sensores galvânicos constam de um conjunto de barras de aço-carbono, eletricamente isoladas. Com o embutimento do sensor no concreto, essas barras ficam posicionadas em diferentes profundidades, sempre menores do que a da armadura. Esse conjunto de barras do sensor são também denominadas de ânodos [7]. 
VALDÉS, A. C.; ROQUE, P. J.; MEDEIROS, M. H. F., SENSORES DE CORROSÃO PARA MONITORAMENTO DE PONTES DE CONCRETO ARMADO - TESTES INICIAIS. $2^{\circ}$ Simpósio Paranaense de Patologia das Construções (2० SPPC), artigo 2SPPC1012, pp. 129-143, 2017. DOI: 10.4322/2SPPC.2017.012

Um dos sensores galvânicos comercializados é o Sensor de múltiplos elétrodos (CorroWatch Multisensor), da empresa Force Technology; este determina o potencial de circuito aberto das barras do ânodo com o uso do elétrodo de referência ERE20 ( $\left.\mathrm{Mn} / \mathrm{MnO}_{2}\right)$ embutido na sua proximidade, e a temperatura do concreto por meio de elétrodo específico embutido na base do sensor [8]. Usualmente, a avaliação do risco de corrosão é feita com a determinação da corrente galvânica e do potencial de circuito aberto.

\section{Materiais e métodos}

\subsection{Configuração do sensor}

A macro célula galvânica considerada neste estudo é formada por cobre e aço carbono dispostos num formato semelhante ao sensor galvânico CorroWhatch da Force Technology. Usaram-se barras de aço-carbono de $60 \mathrm{~mm}$ de comprimento e diâmetro 8-10 mm (ânodo) a diferentes alturas e instalados sobre uma placa de cobre (cátodo) de 50 por $50 \mathrm{~mm}$ e 3-4 mm de espessura. Nas conexões elétricas foi utilizado cabo de cobre isolado com filme de PVC soldado aos metais envolvidos de $2,5 \mathrm{~mm}^{2}$. As barras de aço carbono foram fixadas na placa de cobre com resina para solda em frio. Todos os metais foram polidos com escova com fios de aço. Em seguida, foram enxaguados com água destilada, imersos em álcool e secados ao ar. Ver Figura 1.

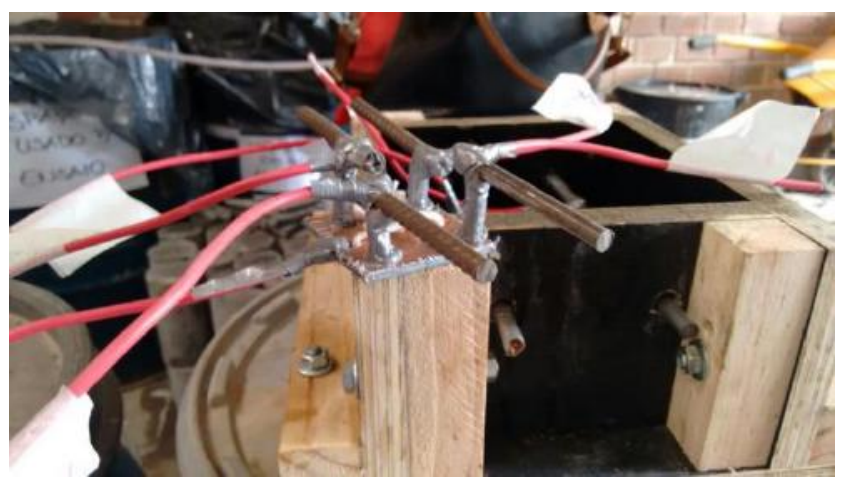

Figura 1: Sensor galvânico. Fonte: Autores

\subsubsection{Influência da área dos eletrodos na estabilidade de carga no sensor}

A diferença de potencial ( $E$ par) média será o potencial de referência do sensor galvânico em estado estacionário. Teoricamente o sensor galvânico deve ser capaz de mantê-lo de forma tal que possa ser usado como um potencial de referência confiável. O potencial de um par $(E$ par $)$ é resultado da combinação dos potenciais dos metais envolvidos e determinado pelo ponto onde a corrente anódica ou de oxidação é igual à corrente catódica ou de redução; esta condição leva à polarização de ambos os metais para um potencial combinado $(E$ par $)$ e assegura a estabilidade deste último no tempo [10]. As correntes anódicas e catódicas estarão em função da área do ânodo e da área de cátodo respectivamente. Por exemplo, para a mesma densidade de corrente um ânodo de $1 \mathrm{~cm}^{2}$ vai produzir menos corrente total que um ânodo de $10 \mathrm{~cm}^{2}[6]$.

Entretanto, teoricamente o ânodo e o cátodo podem ser feitos de qualquer tamanho já que a relação de área entre o ânodo e o cátodo é a maior responsável por manter 
VALDÉS, A. C.; ROQUE, P. J.; MEDEIROS, M. H. F., SENSORES DE CORROSÃO PARA MONITORAMENTO DE PONTES DE CONCRETO ARMADO - TESTES INICIAIS. $2^{\circ}$ Simpósio Paranaense de Patologia das Construções (2० SPPC), artigo 2SPPC1012, pp. 129-143, 2017. DOI: 10.4322/2SPPC.2017.012

um potencial estável do que a área dos eletrodos propriamente dito [10]. Desta forma o período durante o qual o potencial do par se mantém estável pode ser otimizado mudando a relação de área entre o cátodo e o ânodo. Dito período é reconhecido como o tempo de vida útil do sensor galvânico [6].

Tendo em conta tudo o explicado anteriormente, a área da placa de cobre que atua como cátodo no sensor galvânico projetado no presente trabalho foi desenhada de maneira que fosse igual à somatória das áreas dos ânodos que compõem a macro célula galvânica (Ver Tabela 1).

Tabela 1: Relação entre as áreas.

\begin{tabular}{ccc}
\hline ÁREA & $\begin{array}{c}\text { VALOR } \\
{\left[\mathbf{m m}^{2}\right]}\end{array}$ & Ac / Aa \\
\hline Área exposta da placa & 2500 & \\
Área dos furos na placa & 942 & 0 \\
Área efetiva do cátodo (Ac) & 1558 & 0,97 \\
Área de um ânodo (Aa) & 1607,68 & \\
\hline
\end{tabular}

\subsubsection{Potencial do par ( $\left.E_{\text {par }}\right)$}

O potencial de um eletrodo ou metal não pode ser obtido empiricamente e não é possível determinar o valor para cada eletrodo no par utilizando o potencial das células galvânicas obtidas empiricamente. Para determinar então o potencial de eletrodo dos metais envolvidos no par usa-se um eletrodo de referência, por exemplo, um eletrodo padrão de hidrogênio.

Os potenciais do eletrodo obtidos por meio deste processo são convencionalmente definidos como potenciais de redução ou potencial de eletrodo padrão, abreviado $\mathrm{E}^{\circ}$; sendo assim, basta reverter o signo do valor obtido se o metal analisado de acordo à série galvânica tem um comportamento ativo (ânodo).

O potencial de circuito aberto de cada um dos metais envolvidos pode-se obter a partir dos correspondentes Diagramas de Pourbaix, mas a posição relativa dos dois metais em uma série galvânica pode variar em função do ambiente.

Finalmente, a base para uma célula eletroquímica, como a célula galvânica é sempre uma reação redox que pode ser dividida em duas semi-reações: oxidação no ânodo (perda de elétrons) e redução no cátodo (ganho de elétrons). Ver Equação 1.

$$
E_{\text {cel }}^{o}=E_{\text {catodo }}^{o}-E_{\text {anodo }}^{o}
$$

De forma tão que se $E^{\circ}$ célula> 0 , então o processo é espontâneo (célula galvânica) e se $\mathrm{E}^{\circ}$ célula< 0 , então o processo é não-espontâneo (célula eletrolítica).

\section{Programa experimental}

Durante a primeira etapa do experimento se analisou o desempenho de dois sensores com a mesma configuração (Ver figura 1): Sensor A e Sensor B. Esses foram sometidos a duas condições de exposição diferentes: - embebidos em uma 
VALDÉS, A. C.; ROQUE, P. J.; MEDEIROS, M. H. F., SENSORES DE CORROSÃO PARA MONITORAMENTO DE PONTES DE CONCRETO ARMADO - TESTES INICIAIS. $2^{\circ}$ Simpósio Paranaense de Patologia das Construções (2० SPPC), artigo 2SPPC1012, pp. 129-143, 2017. DOI: 10.4322/2SPPC.2017.012

solução de $\mathrm{Ca}(\mathrm{OH})_{2}$ simulando a solução intercapilar do concreto em condições normais e com adição de $3,5 \%$ de $\mathrm{NaCl}$ em massa a temperatura ambiente para representar um meio contaminado com íons cloretos -. Foi controlada a Diferença de potencial ( $E$ par) entre os metais envolvidos e a Corrente galvânica (Igal). A solução saturada de $\mathrm{Ca}(\mathrm{OH})_{2}$ foi preparada com água destilada e hidróxido de cálcio P.A Ca $(\mathrm{OH})_{2}$. Este último foi adicionado até que o pH da solução estivesse na faixa de 12 a 13. $\mathrm{O}$ pH da solução e a temperatura ambiente foram controlados antes de cada leitura com um Medidor de pH LUCA (210) com 90 a 100\% de confiabilidade após calibração.

\subsection{Teste do potencial do par galvânico}

Para a avaliação da variação da diferença de potencial do par se usou um adquiridor de dados LabVIEW 8.5. O sensor projetado tem quatro ânodos e cada um deles gera uma leitura individual correspondente a um par ânodo-cátodo. Cada par é reconhecido pelo adquiridor de dados LabVIEW como um canal individual. O software associado ao equipamento registra a diferença de potencial por canal em [V/s]. O valor médio atingido neste estágio foi considerado como o potencial do sensor. Analisou-se a estabilidade da leitura de diferença de potencial no tempo.

\subsection{Teste de corrente galvânica}

A medição da corrente galvânica geralmente se faz com um amperímetro de resistência nula já que a corrente galvânica é da ordem de micros amperes. Por isto, foi usada a técnica eletroquímica Amperímetro de resistência nula (ZRA) do software EC-Lab. no potenciostato SP-200.

A técnica Amperímetro de resistência nula é uma aplicação para a medição de corrente galvânica em um par formado por metais diferentes. É usada também para desempenhar alguns tipos de medições de ruído eletroquímico. Consiste em aplicar zero volts entre o eletrodo de trabalho (WE) e o contra eletrodo (CE) e medir a corrente e o potencial ( $E_{w e}, E_{c e}$ ) versus o eletrodo de referência (REF) [13]. No sensor, os eletrodos de trabalho são as barras de aço carbono e o eletrodo de referência é a placa de cobre.

No teste imerso na solução saturada de hidróxido cálcio contaminada com 3,5\% de cloreto de sódio, os sensores foram extraídos da solução antes de cada leitura para determinar a perda de massa dos ânodos com relação ao peso original. Para isto, se eliminaram os produtos de corrosão da superfície dos ânodos dos sensores enxaguando-os com água destilada; posteriormente foram secados com ar quente por um período de uma hora e finalmente pesados em uma balança com $0,1 \mathrm{mg}$ de precisão.

Os ensaios de Diferença de potencial e de Corrente galvânica foram desenvolvidos em três ocasiões; cada vez se fizeram três leituras. O Esquema 1 mostra o tempo transcorrido entre os conjuntos de leituras. 
VALDÉS, A. C.; ROQUE, P. J.; MEDEIROS, M. H. F., SENSORES DE CORROSÃO PARA MONITORAMENTO DE PONTES DE CONCRETO ARMADO - TESTES INICIAIS. $2^{\circ}$ Simpósio Paranaense de Patologia das Construções (2० SPPC), artigo 2SPPC1012, pp. 129-143, 2017. DOI: 10.4322/2SPPC.2017.012

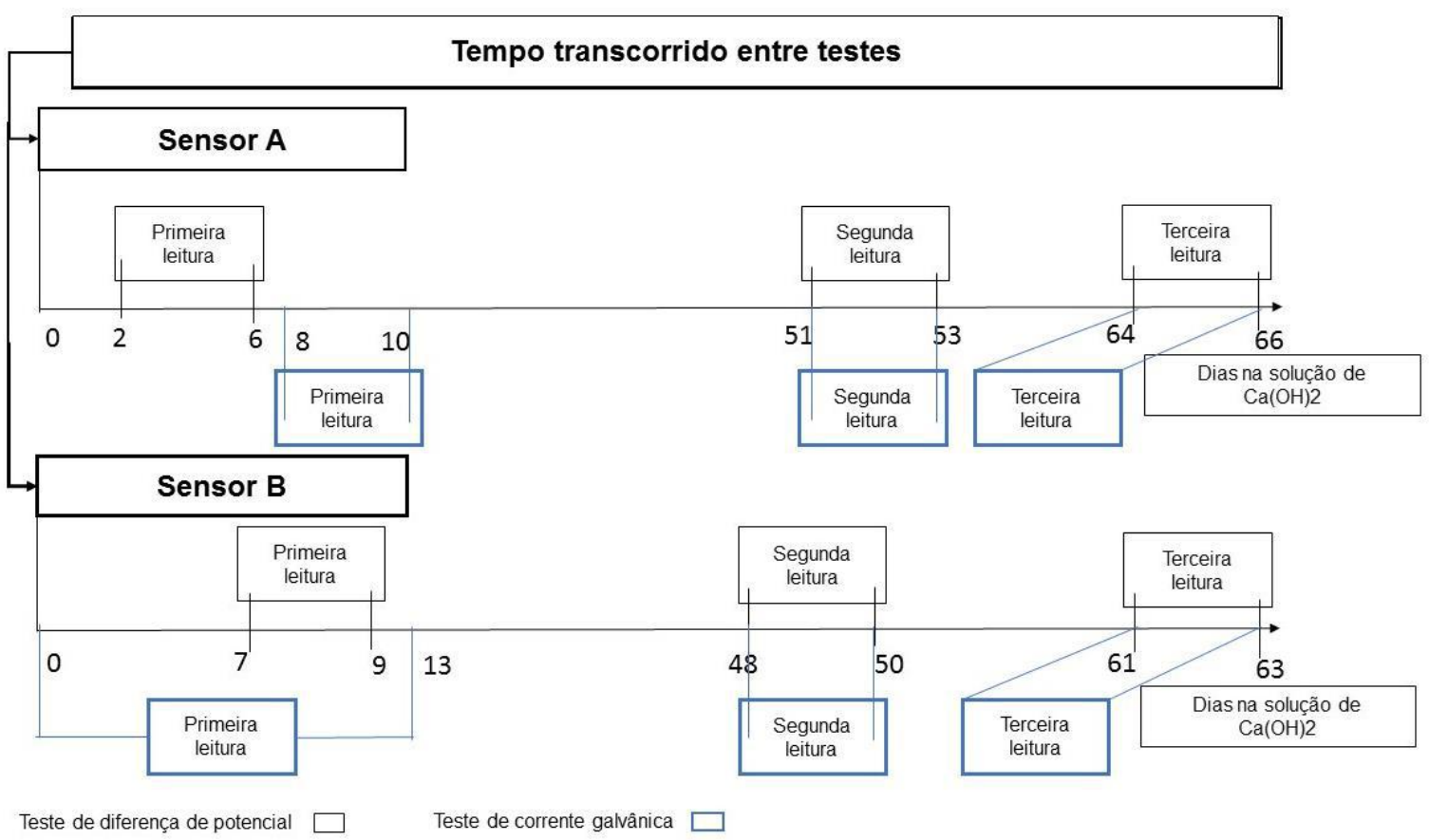

Figura 2: Esquema do experimento

\subsection{Resultados e discussão}

\subsubsection{Teste do potencial do par}

O potencial elétrico varia com a concentração, temperatura e pressão do ambiente no qual está o eletrodo. Sendo assim, não existia forma de conhecer o potencial do sensor formada por aço carbono e cobre em uma solução saturada de hidróxido de cálcio com pH entre 12 e 13 e após ser contaminada com $\mathrm{NaCl}$. Com o intuito de determinar o potencial do par nestas condições foi lida a diferença de potencial dos sensores três vezes por intervalos de 20 minutos a 1 hora; duas leituras antes da contaminação.

No processamento dos dados se fez a conversão das leituras de [V] em [mV]; determinou-se a leitura média em [mV/min.] e para o conjunto de dados resultante se calculou a média aritmética e o desvio padrão para cada um dos pares de maneira individual de forma tal que fosse possível avaliar cada par ânodo-cátodo por separado. Finalmente foi desenvolvido a Analise de variância ANOVA para o sensor no seu conjunto visando definir a origem da variação nas leituras no experimento.

Os gráficos do número 1 ao número 3 resumem o comportamento do Sensor $A$ no período de tempo representado no Esquema 1. Nos gráficos o primeiro par ânodo 1cátodo está representado como ch00, o segundo par ânodo 2 - cátodo como Ch02 e assim sucessivamente até o quarto par do sensor. 
VALDÉS, A. C.; ROQUE, P. J.; MEDEIROS, M. H. F., SENSORES DE CORROSÃO PARA MONITORAMENTO DE PONTES DE CONCRETO ARMADO - TESTES INICIAIS. $2^{\circ}$ Simpósio Paranaense de Patologia das Construções (2० SPPC), artigo 2SPPC1012,

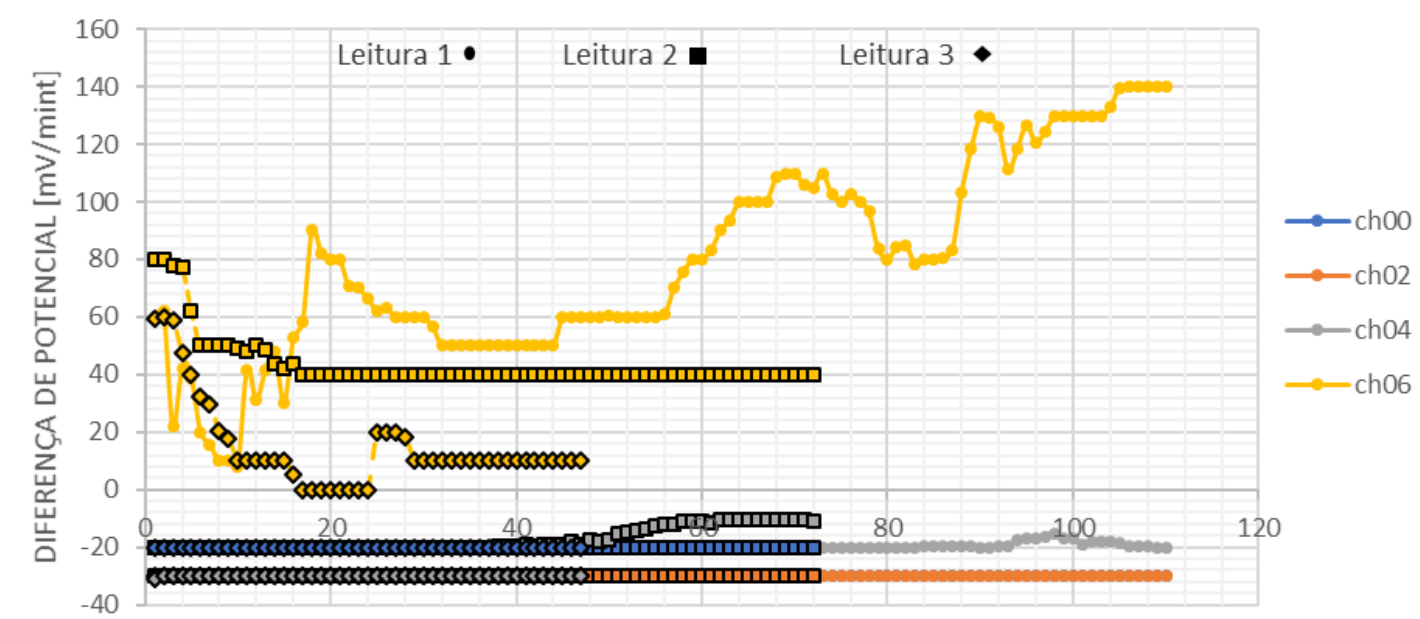

Gráfico 1: Primeiro conjunto de leituras de diferença de potencial. Sensor A

A diferença de potencial dos pares 1, 2 e 3 permaneceu no intervalo de -20 a -30 $\mathrm{mV} / \mathrm{min}$. O potencial do par 1 variou decrescentemente de -30 a $-20 \mathrm{mV} / \mathrm{min}$., do par 3 no sentido contrário e do par 2 se manteve igual a $-30 \mathrm{mV} / \mathrm{min}$. O potencial do par 4, de modo diferente,variou constantemente diminuindo desde a primeira leitura até a terceira sempre na faixa dos valores positivos. Durante a leitura 2 estabilizou-se em $40 \mathrm{mV} / \mathrm{min}$. até o final do intervalo, apresentando menor variação do que na primeira leitura; durante a leitura três, da mesma forma que na segunda, variou no começo do experimento mas termina por se estabilizar em $10 \mathrm{mV} / \mathrm{min}$.

Os valores representados nos gráficos são resultado da combinação dos potenciais do cobre e do aço carbono ( $E_{\text {par }}$. A análise de variância mostrou que teve variação nas leituras entre os quatro pares do sensor e somente o par 2 manteve desvio padrão igual a zero nas três leituras.

A diferença de potencial média em cada leitura foi considerada como o potencial de referência do sensor galvânico em estado estacionário. Teoricamente o sensor galvânico deve ser capaz de manter ou voltar a dita média no transcurso do tempo de forma tal que possa ser usada como um potencial de referência confiável se as condições do meio no qual está o sensor não mudam. Durante esta primeira leitura a média ou potencial de referência do sensor diminuiu.

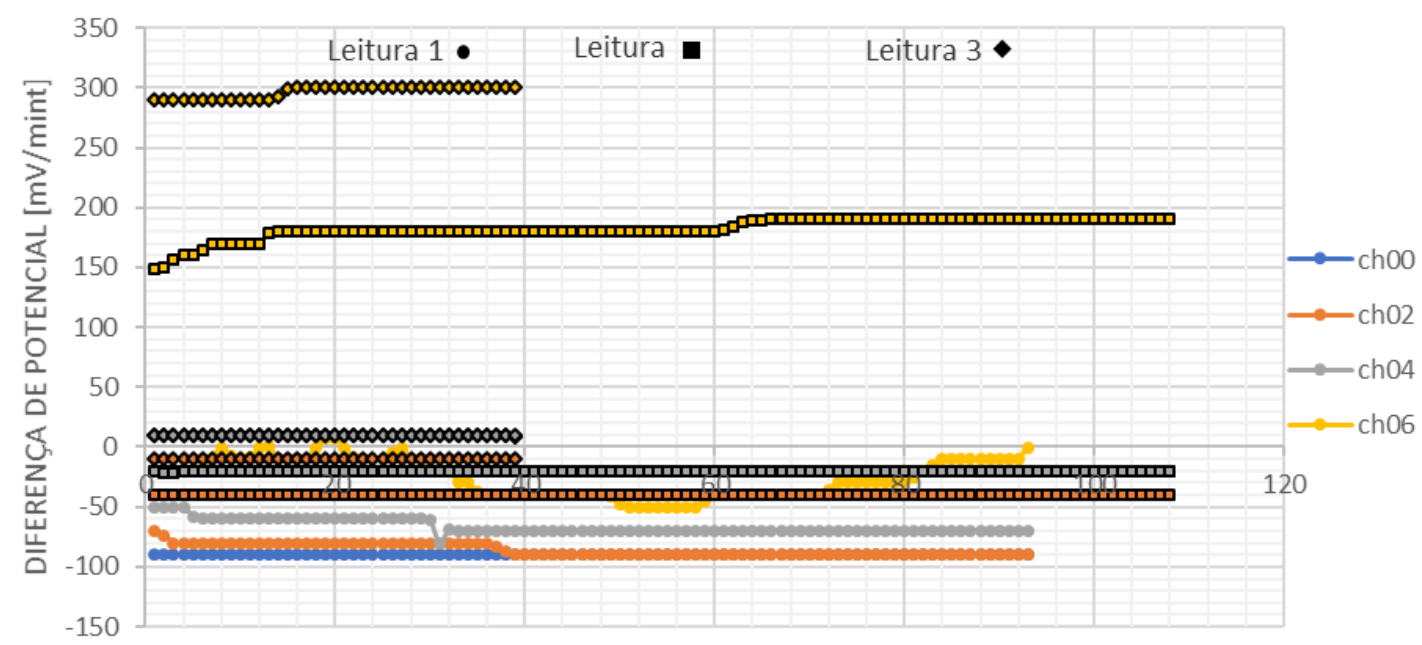

Gráfico 2: Segundo conjunto de leituras de diferença de potencial. Sensor A 
VALDÉS, A. C.; ROQUE, P. J.; MEDEIROS, M. H. F., SENSORES DE CORROSÃO PARA MONITORAMENTO DE PONTES DE CONCRETO ARMADO - TESTES INICIAIS. $2^{\circ}$ Simpósio Paranaense de Patologia das Construções (2० SPPC), artigo 2 SPPC1012, pp. 129-143, 2017. DOI: 10.4322/2SPPC.2017.012

Neste segundo conjunto durante a leitura 1 teve grande variação entre os quatro pares. Acredita-se que esse comportamento foi resultado da queda no valor de $\mathrm{pH}$ da solução saturada, o qual estava no intervalo de 9 a 10 produto do assentamento do hidróxido de cálcio. $\mathrm{O} \mathrm{pH}$ foi corrigido antes de começar a ler a diferença de potencial, mas é possível que esse fato tenha criado instabilidade no meio envolta ao sensor galvânico.

A diferença de potencial dos pares 1,3 e 4 mudou de valores negativos para valores positivos o que não aconteceu durante o primeiro conjunto. O potencial do par 2 se manteve negativo no intervalo de -10 a $-40 \mathrm{mV} / \mathrm{min}$.

O potencial do par 4 variou de -25 a $296 \mathrm{mV} / \mathrm{min}$. em média.O desvio padrão deste par mudou de 34 na leitura 1 do primeiro conjunto para 5 na leitura 3 deste segundo conjunto. O comportamento deste par foi diferenciado com relação aos outros três e os altos valores determinaram que a média ou potencial de referência do sensor aumenta-se. O potencial do sensor galvânico A não ficou estável no transcurso do tempo mostrado no Esquema 1.

O Gráfico 3 mostra como se comportou este parâmetro após a contaminação da solução saturada de hidróxido de cálcio. Este terceiro conjunto está composto por quatro leituras. A leitura de referência (Leitura REF) foi feita 20 minutos antes de adicionar o $\mathrm{NaCl}$ com o objetivo de avaliar se a presença de um agente agressivo provocava uma mudança imediata no potencial dos pares o que de fato aconteceu na leitura 1.Todos os potenciais continuaram positivos e atingiram os valores mais altos na leitura 2.

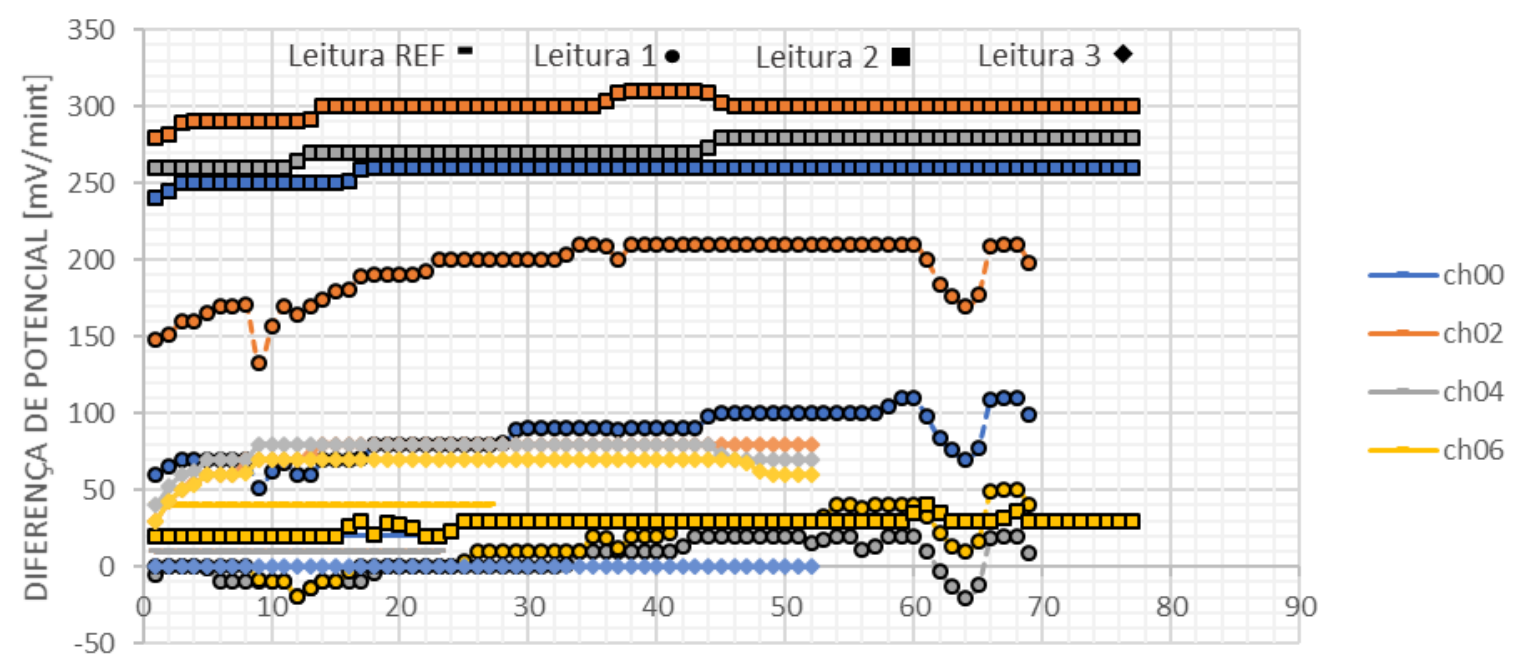

Gráfico 3: Terceiro conjunto de leituras de diferença de potencial. Sensor A

Os gráficos do número 4 ao número 6 resumem o comportamento do Sensor B no período de tempo representado no Esquema 1. 
VALDÉS, A. C.; ROQUE, P. J.; MEDEIROS, M. H. F., SENSORES DE CORROSÃO PARA MONITORAMENTO DE PONTES DE CONCRETO ARMADO - TESTES INICIAIS. $2^{\circ}$ Simpósio Paranaense de Patologia das Construções (2० SPPC), artigo 2SPPC1012, pp. 129-143, 2017. DOI: $10.4322 / 2$ SPPC.2017.012

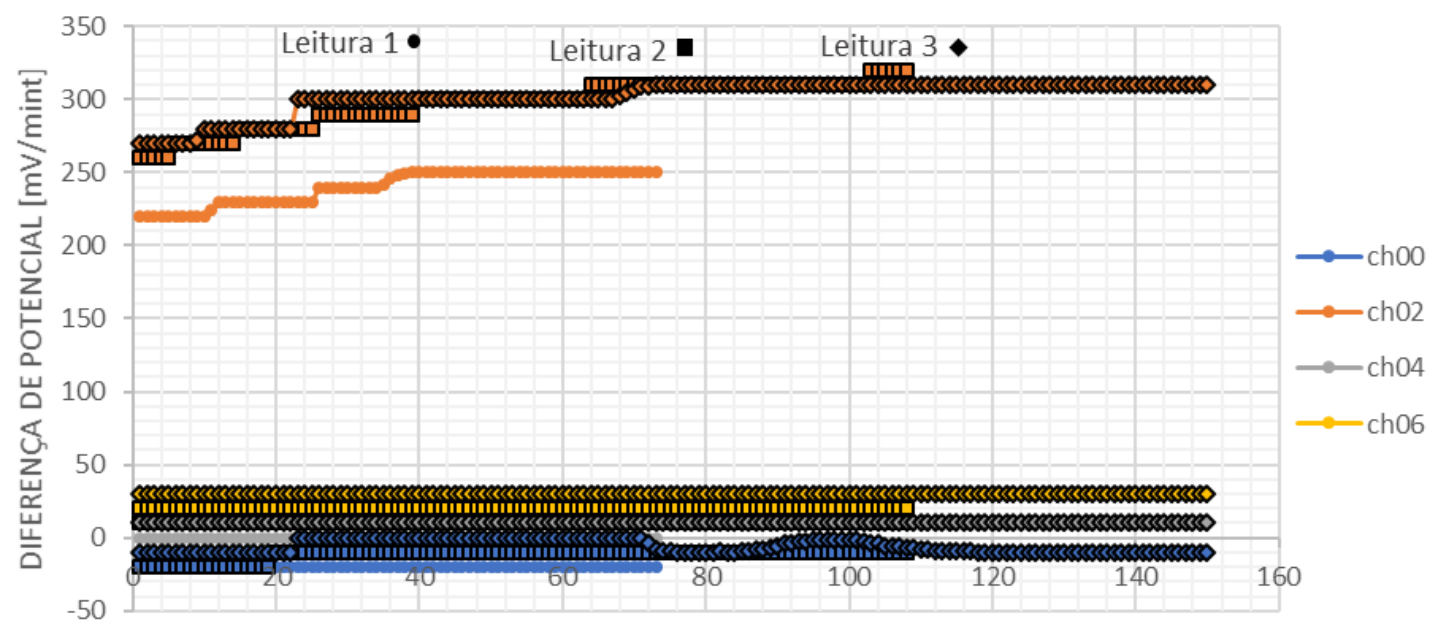

Gráfico 4: Primeiro conjunto de leituras de diferença de potencial. Sensor B

O Sensor B tinha 6 dias na solução de $\mathrm{Ca}(\mathrm{OH})_{2}$ quando foi feita a primeira leitura (Ver Esquema 1), sem embargo observou-se grande variação nas leituras se comparado um par com relação a outro. No Gráfico 4 se observa que o par 1 é o único cuja diferença de potencial foi negativa nas três leituras deste primeiro conjunto. O par 2 manteve o maior potencial dos quatro pares por cima de 250 $\mathrm{mV} / \mathrm{min}$. Os pares 3 e 4 apresentaram maior estabilidade que os primeiros variando de 10 a $35 \mathrm{mV} / \mathrm{min}$.

Dado que a diferença de potencial dos pares 2,3 e 4 é positiva em estado estacionário na presença de íons cloretos a reação entre o cobre e o aço carbono deverá ser espontânea (célula galvânica). Um dos fatores que pode ter determinado este comportamento é a diferença de tempo na solução já que na leitura 3 do segundo conjunto do Sensor A três dos quatro pares apresentaram potenciais positivos (Ver gráfico 2).

Sendo assim, pode-se concluir parcialmente que o potencial de um sensor galvânico se torna positivo no transcurso do tempo se o pH fica estável no intervalo de 12 a 13. Durante esta primeira leitura, a média ou potencial de referência do Sensor $B$ diminuiu da mesma forma que no Sensor $A$.

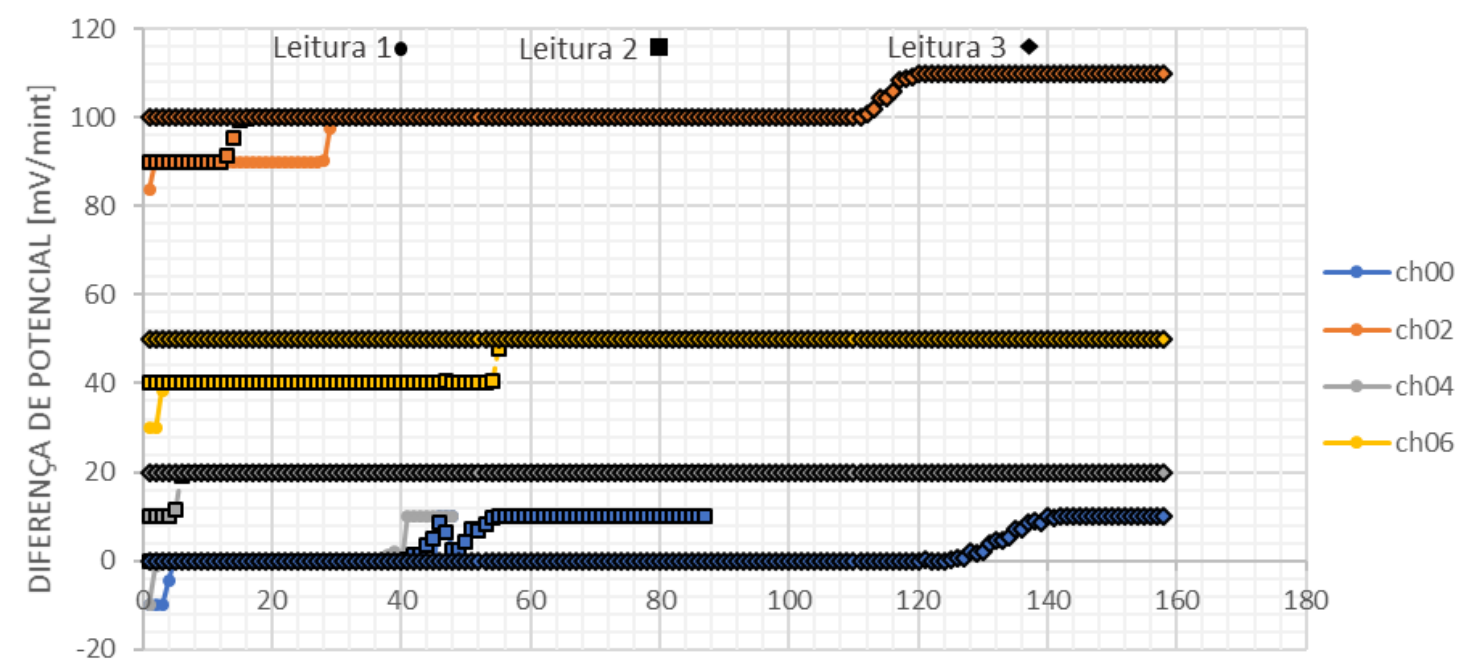

Gráfico 5: Segundo conjunto de leituras de diferença de potencial. Sensor B 
VALDÉS, A. C.; ROQUE, P. J.; MEDEIROS, M. H. F., SENSORES DE CORROSÃO PARA MONITORAMENTO DE PONTES DE CONCRETO ARMADO - TESTES INICIAIS. $2^{\circ}$ Simpósio Paranaense de Patologia das Construções (20 SPPC), artigo 2SPPC1012, pp. 129-143, 2017. DOI: 10.4322/2SPPC.2017.012

$\mathrm{O} \mathrm{pH}$ da solução saturada onde estava submerso este sensor também foi corrigido antes de começar a ler a diferença de potencial, porém o valor registrado pelo LUCADEMA não foi menor do que 11,59. Ainda assim os valores de potenciais de três dos quatro pares na leitura 1 deste segundo conjunto resultaram ser menores do que os valores registrados na última leitura do primeiro conjunto (Ver Gráfico 5).

A diferença de potencial dos pares 2,3 e 4 continuou positiva. O potencial do par 1 virou positivo após a leitura 1. Os pares apresentaram maior estabilidade durante as três leituras. A média ou potencial de referência do Sensor B aumentou durante este segundo conjunto de leituras. O potencial do sensor galvânico B tampouco ficou estável no transcurso do tempo mostrado no Esquema 1. No gráfico 6 ficou representado o comportamento do Sensor B após a contaminação.

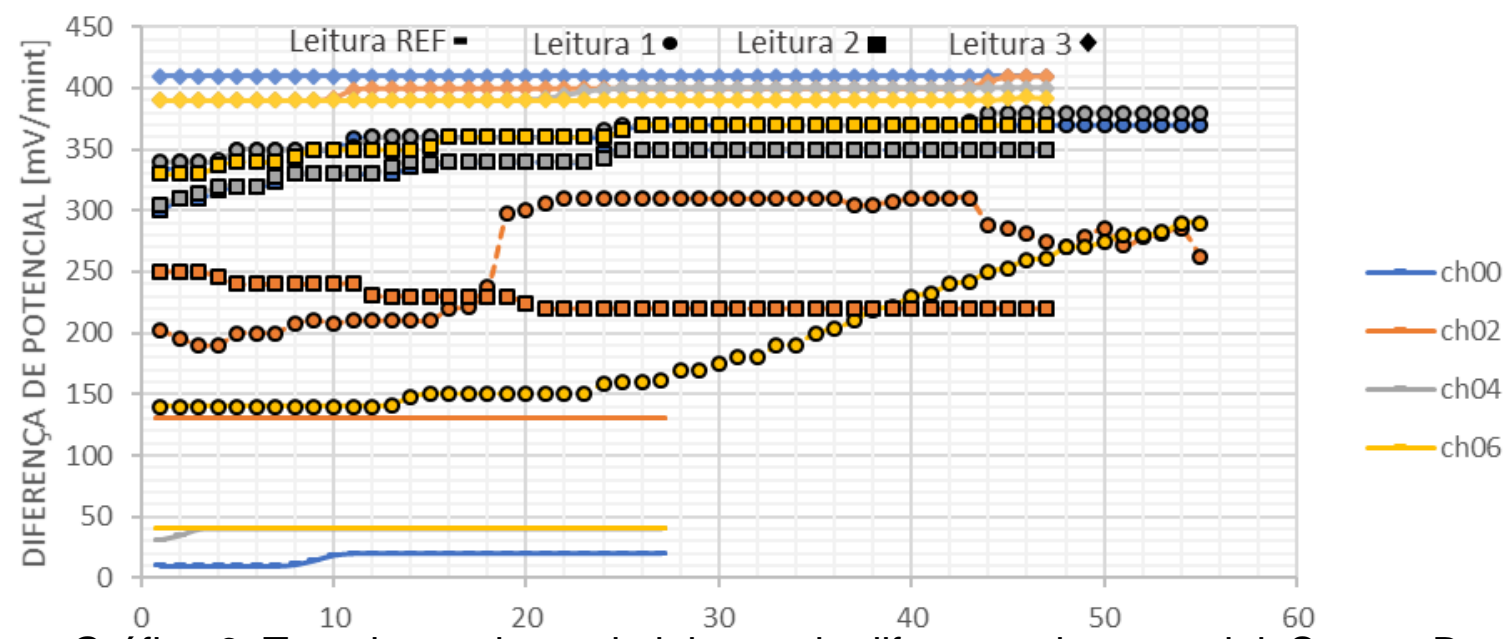

Gráfico 6: Terceiro conjunto de leituras de diferença de potencial. Sensor B

Neste caso os potenciais aumentaram a partir da adição de $\mathrm{NaCl}$ sendo que os valores registrados na leitura 3 foram maiores do que os valores registrados na leitura 1. Durante a leitura de referência (Leitura REF) a variação nas leituras de cada par foi pequena sem embargo imediatamente após a contaminação os potenciais se comportaram de forma instável (Leitura 1).

\subsubsection{Teste de corrente galvânica}

A diferença entre o potencial positivo correspondente ao cátodo e o potencial negativo correspondente ao ânodo gera eletricidade entre os eletrodos da ordem de micros amperes em estado estacionário. Quando o teor de íons cloreto atinge um nível crítico ou que a frente de carbonatação alcança as barras do ânodo do sensor, a eletricidade entre os eletrodos aumenta. No intuito de avaliar o desempenho do sensor desenhado na medição da corrente galvânica em ambas as condições foi lida a corrente galvânica em três espaços de tempo mostrados no Esquema 1. Os gráficos do número 7 ao 9 mostram os resultados de corrente galvânica para 0 Sensor A. 
VALDÉS, A. C.; ROQUE, P. J.; MEDEIROS, M. H. F., SENSORES DE CORROSÃO PARA MONITORAMENTO DE PONTES DE CONCRETO ARMADO - TESTES INICIAIS. $2^{\circ}$ Simpósio Paranaense de Patologia das Construções (2० SPPC), artigo 2SPPC1012, pp. 129-143, 2017. DOI: 10.4322/2SPPC.2017.012

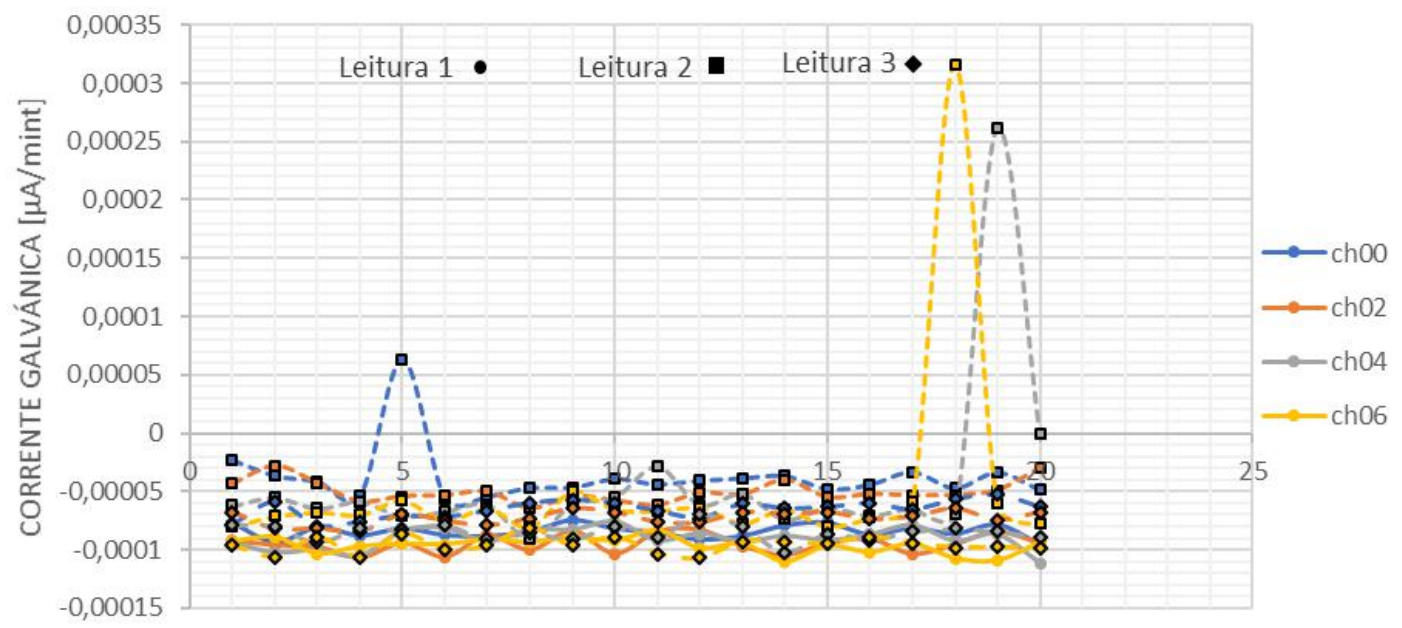

Gráfico 7: Primeiro conjunto de leituras de corrente galvânica. Sensor A

Os valores de corrente galvânica (Igal) ficaram negativos e da ordem de microamperes nas três leituras de ambos conjuntos. O comportamento deste parâmetro foi uniforme nos quatro pares do sensor sempre na faixa dos valores negativos.

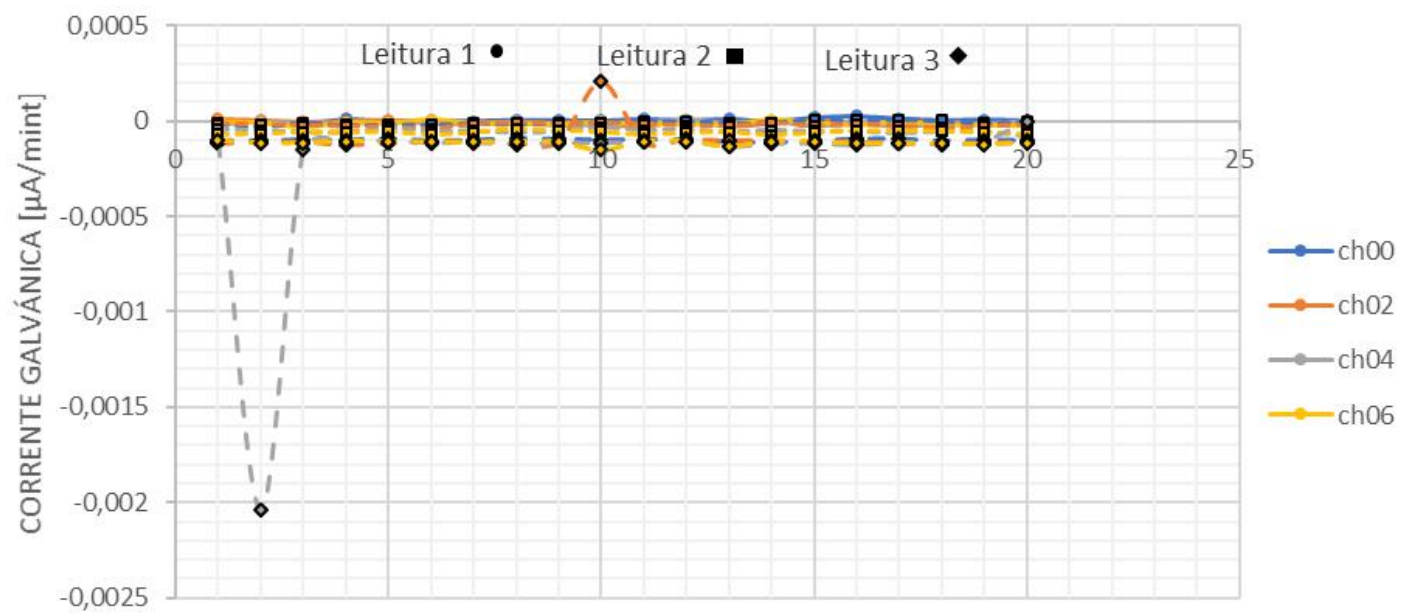

Gráfico 8: Segundo conjunto de corrente galvânica. Sensor A

Os maiores valores foram registrados na última leitura deste segundo conjunto. Esta foi desenvolvida após 9 dias de ter submergido o sensor na solução saturada de hidróxido de cálcio; neste momento o valor de $\mathrm{pH}$ da solução era 12,73. Sendo assim, o fator que deve ter influenciado o aumento das leituras é o tempo imerso na solução.

Os seguintes gráficos mostram o comportamento do Sensor A na solução saturada de hidróxido de cálcio contaminada com 37,35 gramas de $\mathrm{NaCl}$. Este terceiro conjunto de leituras começou quando o sensor tinha 64 dias na solução (Ver Esquema 1). Antes de cada leitura foi registrada a massa do sensor com o intuito de controlar a perda de massa dos ânodos. 
VALDÉS, A. C.; ROQUE, P. J.; MEDEIROS, M. H. F., SENSORES DE CORROSÃO PARA MONITORAMENTO DE PONTES DE CONCRETO ARMADO - TESTES INICIAIS. $2^{\circ}$ Simpósio Paranaense de Patologia das Construções (2० SPPC), artigo 2SPPC1012, pp. 129-143, 2017. DOI: 10.4322/2SPPC.2017.012

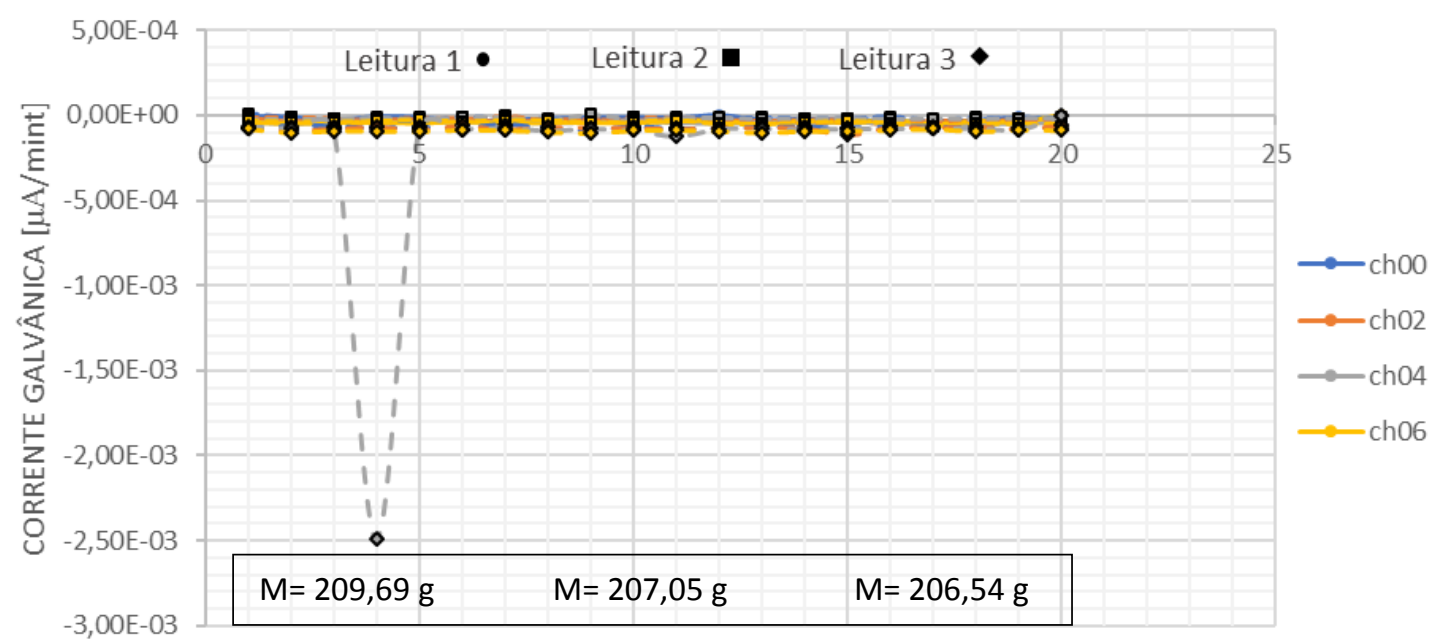

Gráfico 9: Terceiro conjunto de leituras de corrente galvânica. Sensor A

Os valores de massa indicam que os ânodos perderam massa durante as leituras 2 e 3, porém, os dados de corrente não variaram muito com relação às leituras do segundo conjunto (Ver Gráfico 8).

Os gráficos do número 10 ao 12 mostram os resultados de corrente galvânica para o Sensor B antes e após de contaminar a solução de hidróxido de cálcio com $\mathrm{NaCl}$. O gráfico 12 mostra as leituras de corrente com o sensor imerso na solução saturada de hidróxido de cálcio com adição de 37,23 g de $\mathrm{NaCl}$.

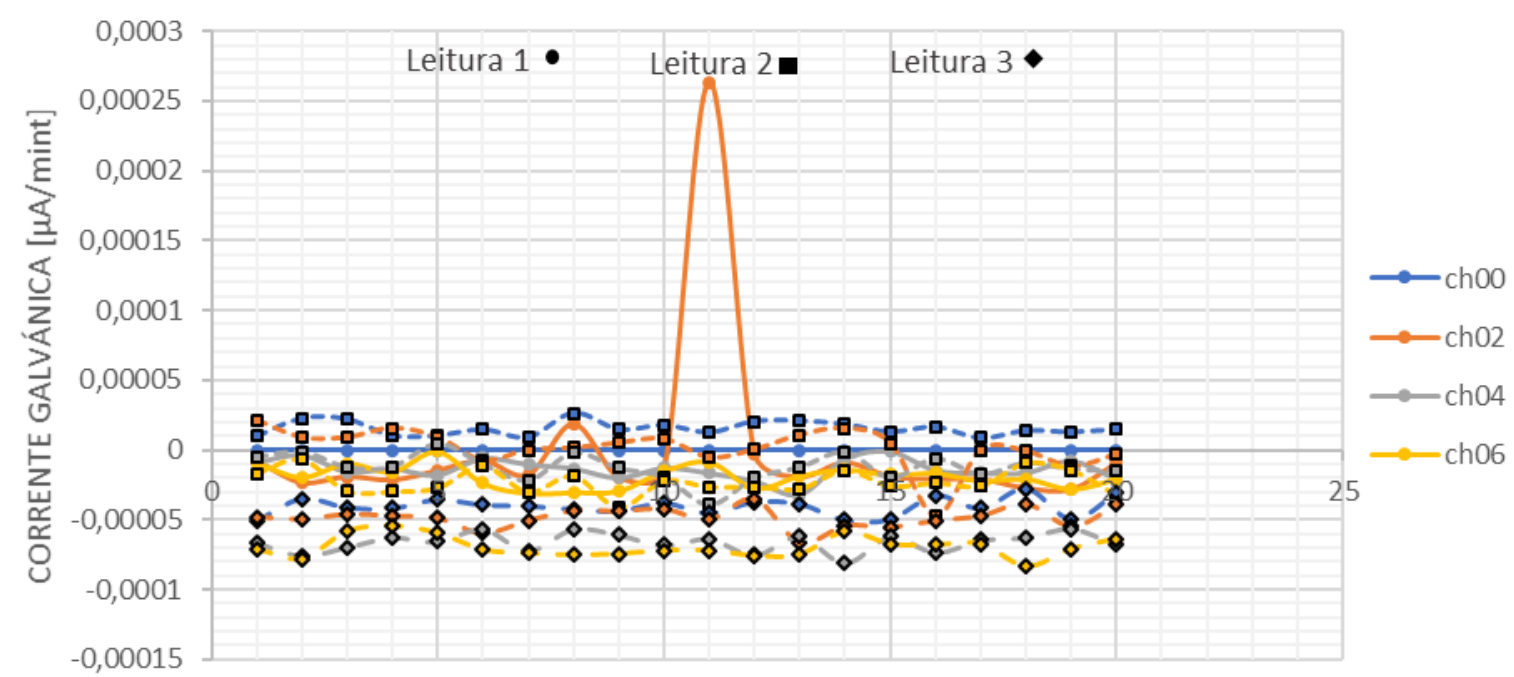

Gráfico 10: Primeiro conjunto de leituras de corrente galvânica. Sensor B

O comportamento do Sensor B durante as leituras 1 e 3 do seu primeiro conjunto foi semelhante ao Sensor A. Destaca-se a leitura 2 porque durante a mesma a corrente gerada nos pares 1 e 2 foi positiva. No segundo conjunto de leituras se destaca a leitura 1 durante a qual a corrente gerada em todos os pares foi positiva. 
VALDÉS, A. C.; ROQUE, P. J.; MEDEIROS, M. H. F., SENSORES DE CORROSÃO PARA MONITORAMENTO DE PONTES DE CONCRETO ARMADO - TESTES INICIAIS. $2^{\circ}$ Simpósio Paranaense de Patologia das Construções (2० SPPC), artigo 2SPPC1012, pp. 129-143, 2017. DOI: 10.4322/2SPPC.2017.012

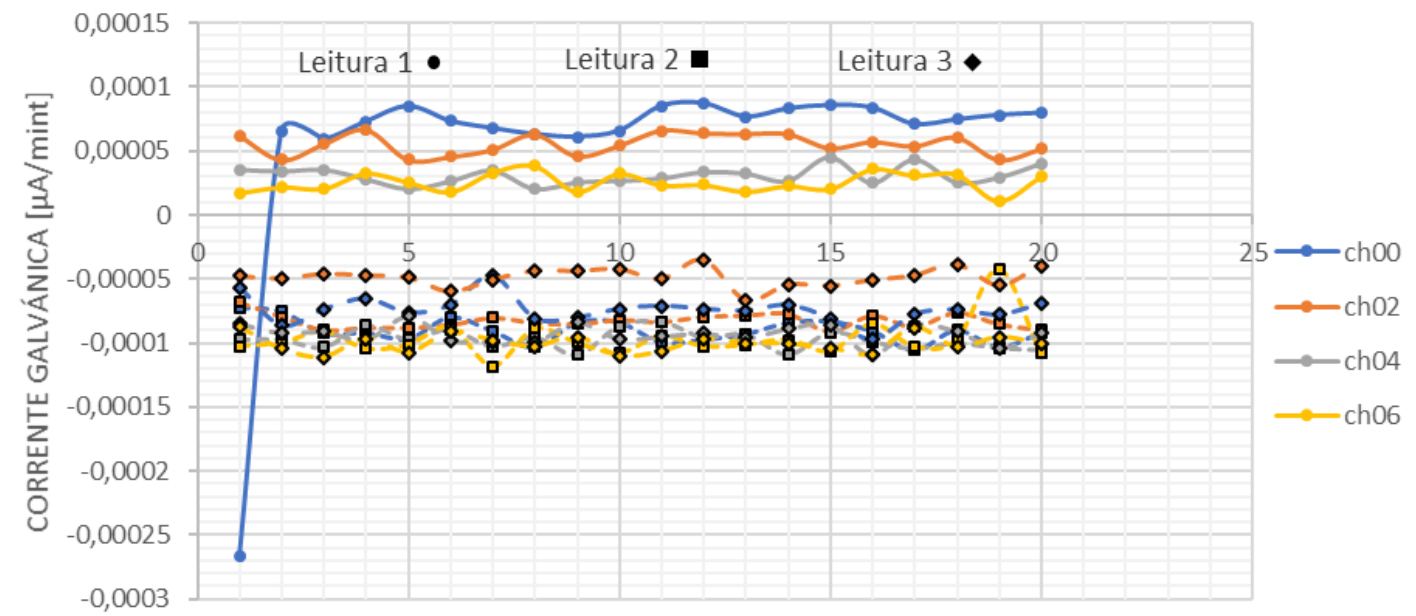

Gráfico 11: Segundo conjunto de leituras de corrente galvânica. Sensor B

As leituras de ambos sensores resultaram ser muito baixas, quase desprezíveis em condições estacionarias. Apesar da variação do pH pelo assentamento do hidróxido de cálcio durante o tempo transcorrido entre o primeiro conjunto de leituras e o segundo, os valores de corrente não sofreram muita variação.

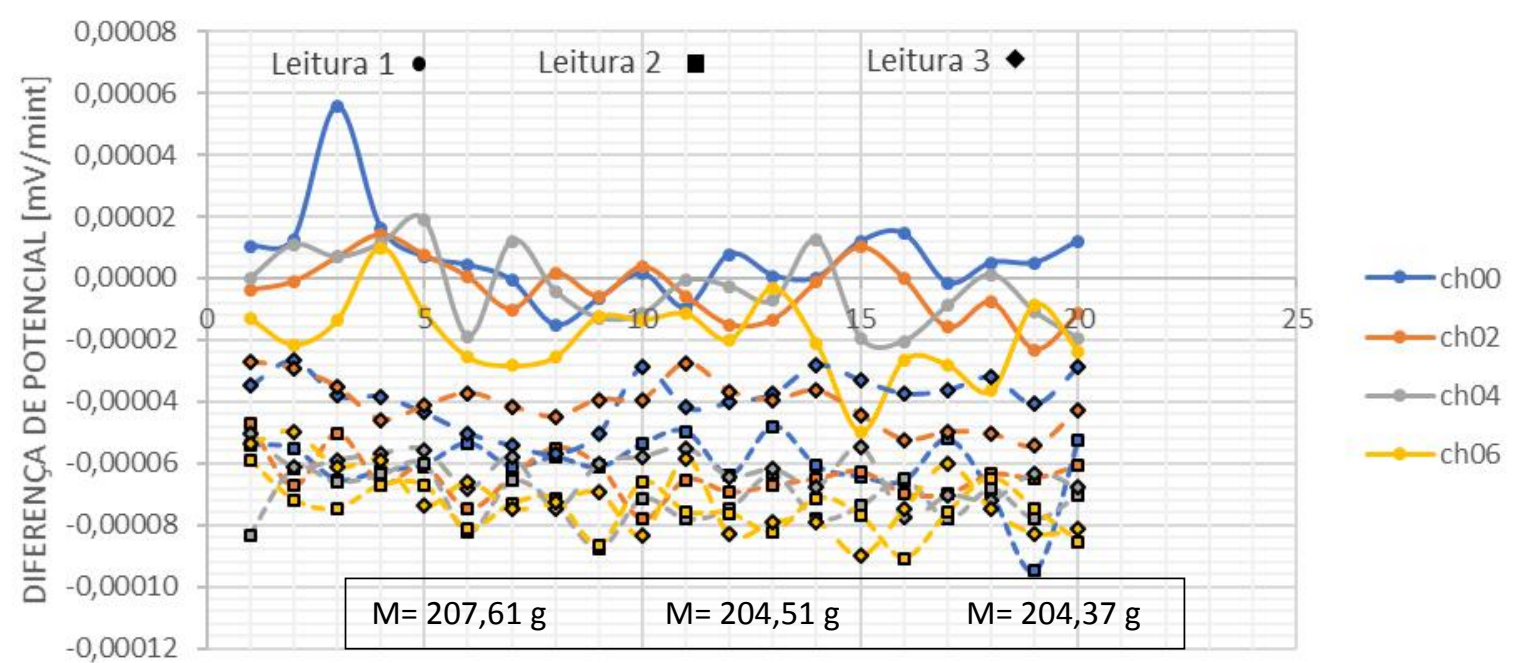

Gráfico 12: Terceiro conjunto de leituras de correntes galvânicas. Sensor B

Com exceção da leitura de corrente gerada no par 1, as leituras restantes foram negativas (Ver Gráfico 12). As leituras de corrente galvânica em ambos os sensores ainda após da adição de cloreto à solução saturada de hidróxido de cálcio continuaram sendo muito baixas. É provável que este comportamento seja resultado do alto valor de $\mathrm{pH}$ da solução, o qual ficou acima de 12 ainda durante o terceiro conjunto de leituras.

\section{Conclusões}

Baseado na teoria eletroquímica o potencial de um sensor galvânico é resultado da diferença de potencial entre o cátodo e o ânodo (Equação 1) de forma tal que o sensor se comporte como um semi-eletrodo de referência capaz de manter ou voltar a um valor médio de potencial no transcurso do tempo. No período de tempo considerado nesta primeira etapa (Ver Esquema 1), nas condições de exposição descritas, os sensores confeccionados não conseguiram manter um valor de 
VALDÉS, A. C.; ROQUE, P. J.; MEDEIROS, M. H. F., SENSORES DE CORROSÃO PARA MONITORAMENTO DE PONTES DE CONCRETO ARMADO - TESTES INICIAIS. $2^{\circ}$ Simpósio Paranaense de Patologia das Construções (2० SPPC), artigo 2 SPPC1012, pp. 129-143, 2017. DOI: 10.4322/2SPPC.2017.012

potencial estável. Apesar disso, no futuro serão necessárias outras leituras para melhor compreensão do comportamento do potencial elétrico do sensor na presença de agentes agressivos.

A variação do $\mathrm{pH}$, o transcorrer do tempo e a presença de íons cloretos provocaram variações no valor de potencial dos pares e a corrente galvânica gerada neles. $\mathrm{Na}$ presença de íons cloretos, o potencial elétrico aumenta inicialmente. No caso de que o pH se torne menor do que 12 este parâmetro diminui. Inicialmente o potencial do sensor foi negativo (reação eletrolítica), mas com o transcurso do tempo se tornou positivo (reação galvânica) e a corrente galvânica aumentou.

É aceito em uma fase inicial, enquanto a profundidade dos agentes agressores não tiver atingido a posição do primeiro ânodo, que as correntes geradas entre os ânodos e o cátodo permanecem na gama das correntes típicas do estado passivo $\left(<2 \mu \mathrm{A} / \mathrm{cm}^{2}\right)$. Quando é atingido um nível crítico de cloretos, a corrente galvânica aumenta. No trabalho, as leituras de corrente galvânica, em ambos sensores ainda após a adição de cloreto à solução saturada de hidróxido de cálcio, continuaram sendo muito baixas. É provável que este comportamento seja resultado do alto valor de $\mathrm{pH}$ da solução, o qual ficou acima de 12 ainda durante o terceiro conjunto de leituras.

\section{Agradecimentos}

Os autores deste trabalho agradecem ao apoio da Fundação Araucária e CNPq, pelo apoio financeiro na forma de bolsa e aquisição de equipamentos empregados na pesquisa. Também registramos um agradecemos especial ao técnico do Laboratório Ricardo Cezar Volert pela enorme ajuda com a instalação e programação do adquiridor de dados.

\section{Referências}

[1] NACE 3T199 (1999) Techniques For Monitoring Corrosion And Related Parameters In Field Applications.

[2] Andrade, Carmen; Félix, Carlos; Figueiras, Helena; Coutinho, Joana Sousa. (2008)Desempenho do kit-sensor de corrosão na monitorização dadurabilidade de estruturas de betão, BE2008 - Encontro Nacional Betão Estrutural.

[3] $\varnothing$. Vennesland; M. Raupach; C. Andrade (2007) Recommendation of Rilem TC 154-EMC: "Electrochemical techniques for measuring corrosion in concrete"measurements with embedded probes, Materials and Structures 40:745-758.

[4] NDT\& E INTERNATIONAL. M. Raupach and P. Schiessl, "Macrocell sensor systems for monitoring of the corrosion risk of the reinforcement in concrete structures", NDT E INT, 34(6), pp. 435-442.

[5] Pereira, Elsa Vaz; M. Manuela Salta (2012) Monitorização permanente da corrosão em estruturas de betão armado. Resultados a longo prazo, Encontro Nacional BETÃO ESTRUTURAL - BE2012.

[6] Pawlick, L.A.; Stoner, Glen E.; Clemeña, Gerardo G. (1998) Development of na embeddable reference electrode for reinforced concrete structures, Virginia transportation research council. 
VALDÉS, A. C.; ROQUE, P. J.; MEDEIROS, M. H. F., SENSORES DE CORROSÃO PARA MONITORAMENTO DE PONTES DE CONCRETO ARMADO - TESTES INICIAIS. $2^{\circ}$ Simpósio Paranaense de Patologia das Construções (2० SPPC), artigo 2 SPPC1012, pp. 129-143, 2017. DOI: 10.4322/2SPPC.2017.012

[7] Araújo, D. Adriana; Panossian, Zehbour; Portella, Pedro D.; Bässler, Ralph (2013) Monitoramento da corrosão em estruturas de concreto: sensor galvânico, Revista Pini.

[8] FORCE Technology - Catalogue for Products related to Corrosion Monitoring in Concrete,

[9] De Lacerda, Marianna Mousinho; Müller, Renata (2015) Uso do sensor de taxa de corrosão instantânea como técnica de monitoramento da corrosão em estruturas de concreto.

[10] https://sites.google.com/site/scientiaestpotentiaplus/corrosao/corrosaogalvanica, Corrosão galvânica, O mecanismo de corrosão conduzido pela formação de pilhas eletroquímicas.

[11] Yoo, Ji-Hong;Parka, Zin-Taek;Kim, Jung-Gu; Chung, Lan (2003) Development of a galvanic sensor system for detecting the corrosion damage of the steel embedded in concrete structures: Part 1. Laboratory tests to correlate galvanic current with actual damage, Cement and Concrete Research 33 (2003) 2057 2062.

[12] Parka, Zin-Taek; Choia, Seok Yoon; Kima, Jung-Gu; Chung, Lan (2003) Development of a galvanic sensor system for detecting the corrosion damage of the steel embedded in concrete structure. Part 2. Laboratory electrochemical testing of sensors in concrete, Cement and Concrete Research 35 (2005) 1814 $-1819$.

[13] EC-Lab Software: Techniques and Applications. Version 10.1x. Bio Logic. Science Instruments (2011). 